\title{
Biomechanical assessment of proximal junctional semi-rigid fixation in long-segment thoracolumbar constructs
}

\author{
Vibhu K. Viswanathan, MBBS, ${ }^{1}$ Ranjit Ganguly, MD, ${ }^{1}$ Amy J. Minnema, MS, ${ }^{1}$ \\ Nicole A. DeVries Watson, PhD, ${ }^{4}$ Nicole M. Grosland, PhD, ${ }^{2-4}$ Douglas C. Fredericks, BS, ${ }^{2}$ \\ Andrew J. Grossbach, MD, ${ }^{1}$ Stephanus V. Viljoen, MD, ${ }^{1}$ and H. Francis Farhadi, MD, PhD ${ }^{1}$

\begin{abstract}
1Department of Neurological Surgery, The Ohio State University Wexner Medical Center, Columbus, Ohio; and Departments of ${ }^{2}$ Orthopaedics and Rehabilitation and ${ }^{3}$ Biomedical Engineering, and ${ }^{4}$ Center for Computer Aided Design, University of lowa, lowa City, lowa
\end{abstract}

\begin{abstract}
OBJECTIVE Proximal junctional kyphosis (PJK) and failure (PJF) are potentially catastrophic complications that result from abrupt changes in stress across rigid instrumented and mobile non-fused segments of the spine (transition zone) after adult spinal deformity surgery. Recently, data have indicated that extension (widening) of the transitional zone via use of proximal junctional (PJ) semi-rigid fixation can mitigate this complication. To assess the biomechanical effectiveness of 3 semi-rigid fixation constructs (compared to pedicle screw fixation alone), the authors performed cadaveric studies that measured the extent of PJ motion and intradiscal pressure changes ( $\triangle I D P)$.
\end{abstract}

METHODS To measure flexibility and $\triangle I D P$ at the PJ segments, moments in flexion, extension, lateral bending (LB), and torsion were conducted in 13 fresh-frozen human cadaveric specimens. Five testing cycles were conducted, including intact (INT), T10-L2 pedicle screw-rod fixation alone (PSF), supplemental hybrid T9 Mersilene tape insertion (MT), hybrid T9 sublaminar band insertion (SLB1), and hybrid T8/T9 sublaminar band insertion (SLB2).

RESULTS Compared to PSF, SLB1 significantly reduced flexibility at the level rostral to the upper-instrumented vertebral level (UIV+1) under moments in 3 directions (flexion, LB, and torsion, $p \leq 0.01)$. SLB2 significantly reduced motion in all directions at UIV+1 (flexion, extension, LB, torsion, $p<0.05$ ) and at $\mathrm{UIV}+2$ (LB, torsion, $p \leq 0.03$ ). MT only reduced flexibility in extension at UIV+1 $(p=0.02)$. All 3 constructs revealed significant reductions in $\triangle I D P$ at UIV+1 in flexion (MT, SLB1, SLB2, $p \leq 0.02$ ) and torsion (MT, SLB1, SLB2, $p \leq 0.05$ ), while SLB1 and SLB2 significantly reduced $\Delta I D P$ in extension (SLB1, SLB2, $p \leq 0.02$ ) and SLB2 reduced $\triangle I D P$ in LB $(p=0.05)$. At UIV+2, SLB2 similarly significantly reduced $\triangle \mathrm{IDP}$ in extension, $\mathrm{LB}$, and torsion ( $\mathrm{p} \leq 0.05)$.

CONCLUSIONS Compared to MT, the SLB1 and SLB2 constructs significantly reduced flexibility and $\triangle I D P$ in various directions through the application of robust anteroposterior force vectors at UIV+1 and UIV+2. These findings indicate that semi-rigid sublaminar banding can most effectively expand the transition zone and mitigate stresses at the PJ levels of long-segment thoracolumbar constructs.

https://thejns.org/doi/abs/10.3171/2018.7.SPINE18136

KEYWORDS proximal junctional kyphosis; proximal junctional failure; sublaminar band; Mersilene tape; spinal deformity; thoracic

$\mathrm{P}$ ROXIMAL junctional kyphosis (PJK) and proximal junctional failure (PJF) are well-known complications following long-segment instrumented adult spinal deformity (ASD) surgery. PJK criteria include focal segmental kyphosis of $10^{\circ}$ or more and a relative $10^{\circ}$ or more kyphotic increase between the upper instrumented vertebra (UIV) and 2 levels cranial to the UIV (UIV+2). ${ }^{7}$ With an incidence of 5.6\%-21\%, ${ }^{11,16} \mathrm{PJF}$ is the most common cause for ASD revision surgery. ${ }^{24}$ Despite the morbidity associated with PJK and PJF, the factors underlying

ABBREVIATIONS ASD = adult spinal deformity; $\mathrm{BMD}=$ bone mineral density; $\mathrm{CT}=$ computed tomography; INT = intact; $\mathrm{LB}=$ lateral bending; $\mathrm{MT}=\mathrm{Mersilene}$ tape; $\mathrm{PJ}=$ proximal junctional; PJF = PJ failure; PJK = PJ kyphosis; PSF = pedicle screw fixation; SLB1 = sublaminar band at UIV+1; SLB2 = sublaminar band at UIV+1 and UIV+2; UIV = upper instrumented vertebra; $\triangle \mathrm{IDP}=$ change in intradiscal pressure.

SUBMITTED February 19, 2018. ACCEPTED July 11, 2018.

INCLUDE WHEN CITING Published online November 2, 2018; DOI: 10.3171/2018.7.SPINE18136. 
their development have not been completely defined., ${ }^{4,5}$ However, data indicate that the abrupt change in stress between the rigid instrumented vertebral segments and the adjacent mobile vertebrae represents the most critical biomechanical factor in the development of PJK and PJF. ${ }^{3}$

To reduce the development of PJK and PJF after longsegment instrumented fusion, semi-rigid implants connecting the proximal portion of the instrumented fusion to the non-instrumented spine have been developed. These constructs are designed to widen the transition zone and reduce stress across this region., ${ }^{1,226,30}$ One proposed model involves the use of posterior tethers proximal to the UIV. ${ }^{2}$ Specifically, augmentation of the posterior spinal ligamentous complex using either spinous process-anchored Mersilene tape (MT; Ethicon) ${ }^{30}$ or polyester sublaminar bands ${ }^{27}$ positioned at the PJ levels and fastened to the long-segment construct has been employed to generate semi-rigid fixation with distinct force vectors. These hybrid constructs are designed to provide a smoother transition and to reduce the stress across the proximal junction, thereby preventing proximal flexion failure. ${ }^{20}$

While these semi-rigid constructs have been employed clinically to potentially reduce PJK and PJF, the comparative biomechanical advantages of each of these constructs have not been defined. To characterize the biomechanical properties of various designs (compared to rigid instrumented fixation), we performed a cadaveric study examining a combination of rigid and PJ semi-rigid fixation constructs and determined if these designs impart an advantage against the development of known risk factors for PJK and PJF.

\section{Methods \\ Experimental Study}

Thirteen fresh frozen human thoracolumbar spines (T7-L2) were used in this study (5 female, 8 male; mean age 56.2 years, range 41-66 years). Specimens were screened based on age $(<70$ years), absence of gross coronal or sagittal deformities, absence of lower thoracic bridging osteophytes, and absence of severe disc height loss that would preclude pressure needle placement. Additionally, a lower limit of $0.8 \mathrm{~g} / \mathrm{cm}^{3}$ bone mineral density (BMD) on quantitative computed tomography (CT) was set for inclusion (mean $1.04 \mathrm{~g} / \mathrm{cm}^{3}$, range $0.81-1.17 \mathrm{~g} / \mathrm{cm}^{3}$ ).

\section{Quantitative CT Analysis}

Dual-energy images ( $80 \mathrm{kV}$ and $150 \mathrm{kV})$ were acquired using a CT scanner with an in-plane resolution of $0.3 \times$ $0.3 \mathrm{~mm}$ and $3-\mathrm{mm}$ axial sections. The $150-\mathrm{kV}$ scans were analyzed using ITK-SNAP by segmenting axial slices at approximately mid-vertebral body height while ensuring exclusion of cortical bone. For each specimen, BMD was calculated in Hounsfield units at individual vertebral body levels. The BMD correlation was determined with the Gammex phantom scanned using the same parameters as above. The phantom provided known densities for several materials, including air, cortical bone, and cancellous bone.

\section{Specimen Preparation}

All specimens were stored at $-20^{\circ} \mathrm{C}$ prior to biome- chanical testing. The specimens were thawed to room temperature, and the paravertebral musculature was carefully removed, leaving all ligamentous structures intact. The most cephalad and caudal vertebrae of each specimen were mounted in polyester resin casts (Bondo Corp.). Wood screws were used to help anchor the vertebral bodies within the potting material. To prevent dehydration, the specimens were routinely irrigated with a $0.9 \%$ sodium chloride solution throughout the test period.

\section{Motion Testing}

A servo-hydraulic MTS 858 Bionix II configured with 2 spine gimbals (MTS Corp.) was used to apply unconstrained load application in all 6 degrees of freedom, namely in flexion, extension, lateral bending, and torsion. Intersegmental motion was assessed via specialized markers rigidly affixed to each vertebral level. Each marker consisted of 3 non-collinear infrared light-emitting diodes detectable by an optoelectronic motion analysis system (Optotrak 3020, Northern Digital Inc.).

Specimens were tested using a hybrid multidirectional test protocol. ${ }^{20}$ Initially, intact specimens were loaded to a known moment and the resulting motions were recorded. Thereafter, following the desired intervention (e.g., pedicle \pm hybrid fixation; see Fig. 1), the specimens were rotated to achieve the range of motion exhibited by the intact specimen. This protocol allows for determination of the effect of the surgical intervention on the instrumented and adjacent intervertebral levels.

For the first motion test, the intact thoracolumbar spine segments were tested nondestructively in a random sequence of flexion/extension (x-axis), bilateral torsion (axial rotation) (y-axis), and bilateral lateral bending ( $\mathrm{z}-$ axis) via the moment loading system set to $\pm 4 \mathrm{~N} \times \mathrm{m} .{ }^{18} \mathrm{In}$ subsequent flexibility tests, the specimens were loaded to obtain the same range of motion as that obtained in the intact condition. However, given the number of planned experimental cycles, the maximum moment was limited to $\pm 6 \mathrm{~N} \times \mathrm{m}$ to prevent destructive loading of the specimens. ${ }^{23}$ All tests were repeated for 3 successive loading and unloading cycles, with the data from the third cycle contributing to the computational analyses. Normalized motion values were calculated to control for interspecimen stiffness differences. Motion at the T11-12 to L1-2 levels was averaged and reported as a single value (T11-L2).

Intradiscal pressures at the PJ motion segments T8-9 and T9-10 as well as at T10-11 (UIV motion segment) were measured using Mikro-Cath pressure transducers (Millar Instruments). Transducers were advanced in an anterior to posterior direction to a depth of $15 \mathrm{~mm}$ into adjacent intervertebral discs. At T9-10, dual transducers separated by $5 \mathrm{~mm}$ were positioned, and the average values were reported.

\section{Testing Paradigm}

All specimens underwent testing in 5 sequential cycles.

1. Intact (INT). Spines were tested intact to assess normal ranges of motion prior to fixation.

2. Pedicle screw fixation (PSF). Pedicle screws $(5.5 \times$ 

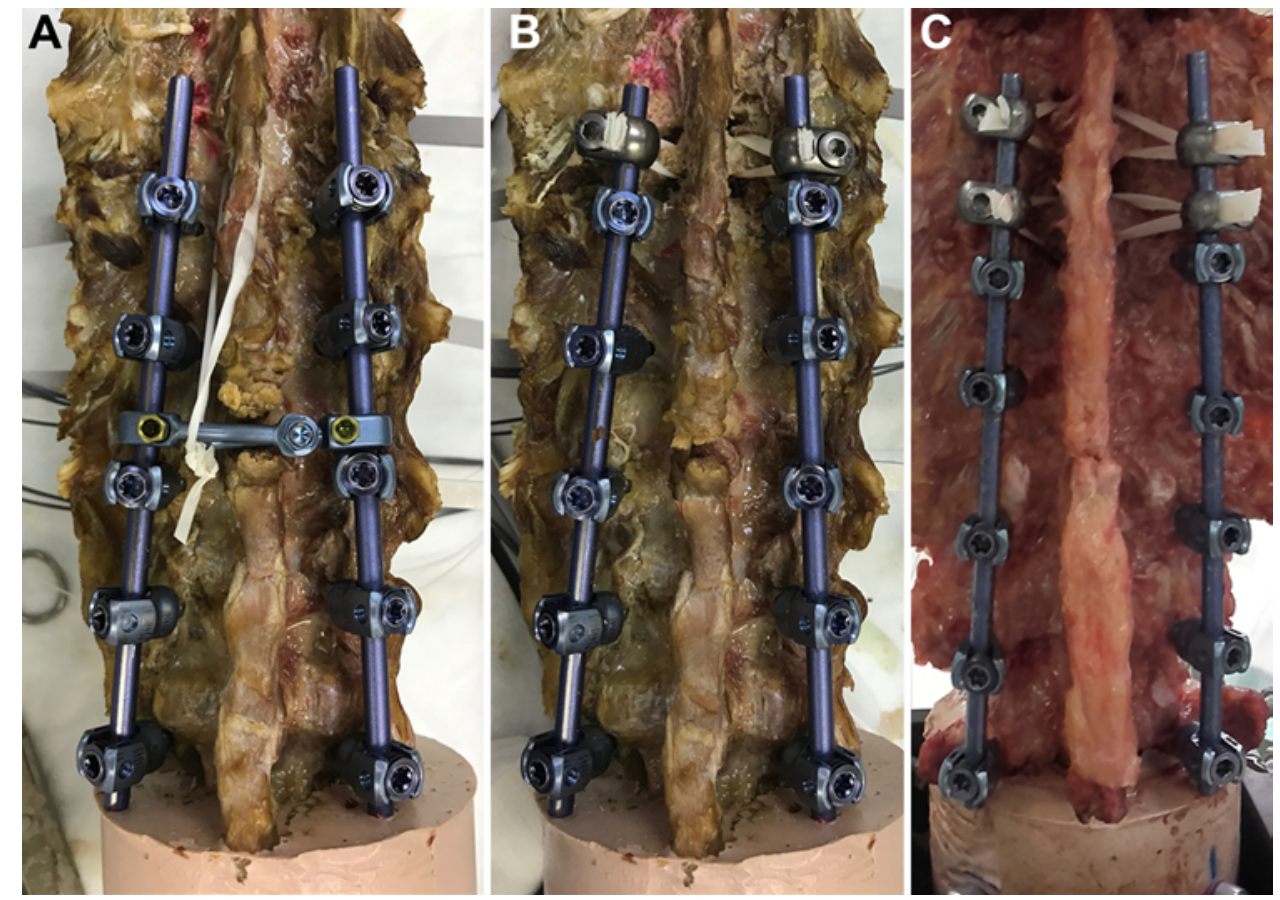

FIG. 1. Photographs of 3 hybrid constructs. A: MT construct. B: SLB1 construct showing placement of bilateral sublaminar bands at the T9 level. C: SLB2 construct showing placement of bilateral sublaminar bands at the T8 and T9 levels.

$45 \mathrm{~mm}$ polyaxial screws; Medtronic) were inserted at the T10, T11, T12, L1, and L2 levels using a free-hand technique with subsequent probing of all the tracts to ensure circumferential walls. Titanium rods were then positioned spanning T10-L2 and firmly fixed within the potting to further ensure rigid caudal fixation.

3. Mersilene tape (MT). A hole was made at the base of the T9 spinous process using a penetrating towel clamp. MT was then passed through the base of the spinous process. The tape was tied to a transverse connector and distraction applied with a rod distractor prior to final tightening (Fig. 1A).

4. Single-level PJ sublaminar band placement at T9 (SLB1). The MT was removed, and bilateral hemilaminotomies were fashioned at T9 and T10. Bilateral sublaminar bands (Jazz; Implanet America Inc.) were placed at the T9 level. The bands were separately attached to the rods, and each band was tensioned using a torquemeter triggered at $2.5 \mathrm{~N} \times \mathrm{m}$ bilaterally (Fig. 1B).

5. Double-level PJ sublaminar band placement at T8 and T9 (SLB2). The previously positioned sublaminar bands were removed, and bilateral hemilaminotomies were fashioned at T8. New bilateral bands were positioned at the T8 and T9 levels. Both bands at each level were separately attached to the rods, and each band was tensioned using a torquemeter triggered at $2.5 \mathrm{~N} \times \mathrm{m}$ (Fig. 1C).

The posterior ligamentous structures and facet joints were kept intact during insertion of the MT and sublaminar bands. The resultant force vectors produced by these hybrid construct designs are shown schematically in Fig. 2; MT insertion and final anchoring results in a rostrocau- dal vector, while final tensioning of the sublaminar bands (SLB1 and SLB2) produces a robust horizontal anteriorto-posterior vector.

\section{Data Analysis}

Load-displacement curves for each spinal level were derived and converted into range of motion ${ }^{19}$ and neutral $z^{2} e^{22}$ for each of the flexibility tests. Intradiscal pressure changes $(\triangle \mathrm{IDP})$ were also compared for each loading paradigm.

\section{Statistical Analysis}

IBM SPSS Statistics v25 (IBM Corp.) was used for the data analysis. Considering the paired and non-parametric characteristics of the study data, Friedman's test (nonparametric ANOVA for repeated measures) was done to compare the overall effect of the configuration groups. In the case of a significant result, pairwise comparisons were performed to assess specific differences between hybrid fixation techniques (MT, SLB1, and SLB2) and PSF. Direct comparisons were made using the Wilcoxon signedrank test with Bonferroni adjustment for 3 comparisons. Median values were compared after exclusion of major outliers. A p value $\leq 0.05$ was considered statistically significant.

\section{Results}

\section{Biomechanical Testing Characteristics}

All the specimens $(n=13)$ underwent the first 4 cycles of testing (INT, PSF, MT, and SLB1), and 9 specimens underwent a further fifth cycle (SLB2). For each speci- 

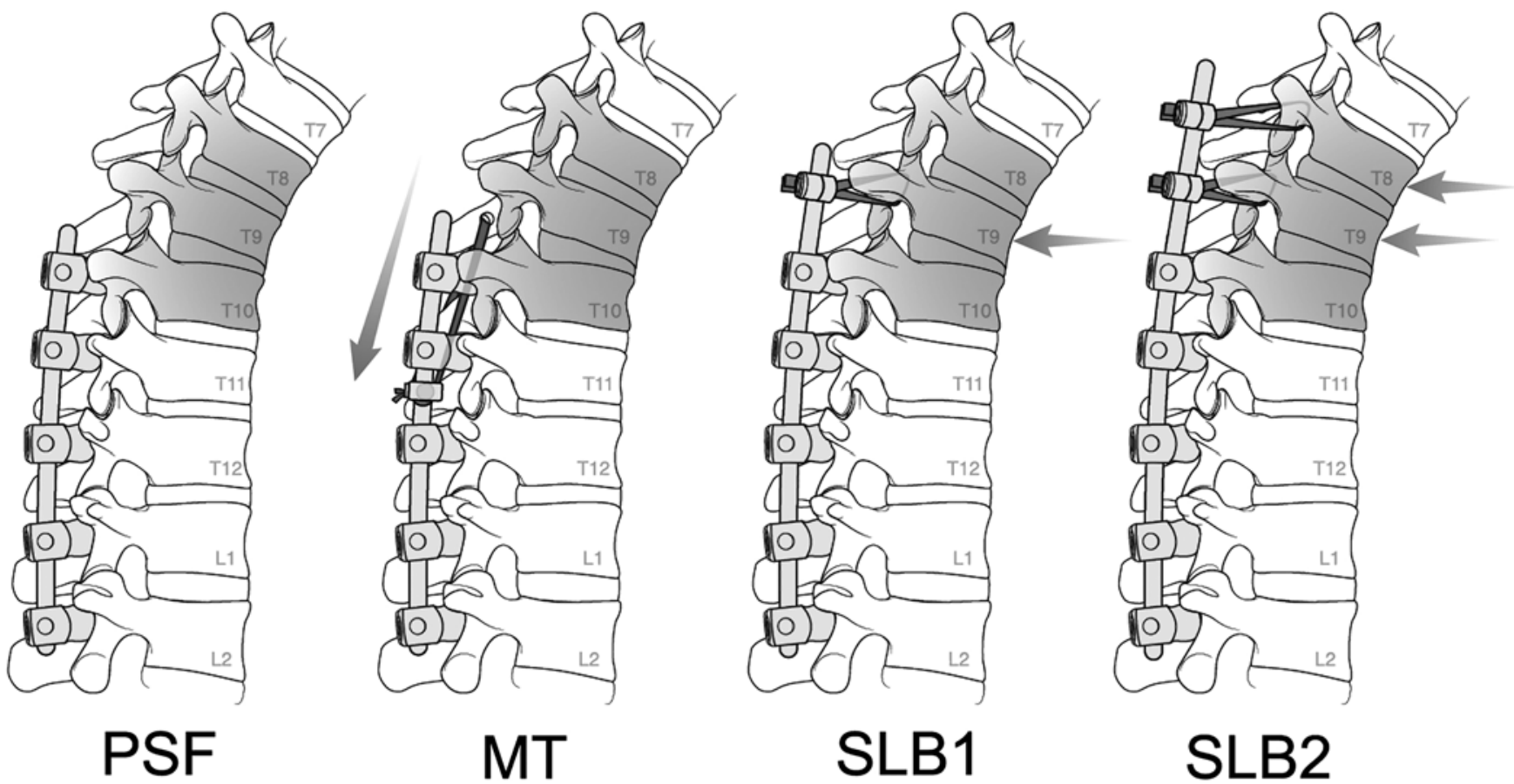

MT

SLB2

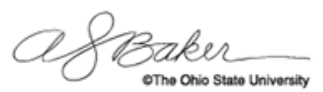

FIG. 2. Schematic diagram of PSF and the 3 hybrid constructs (MT, SLB1, and SLB2). The functional spinal units at the proximal junction exposed to high stress are shaded in gray. The MT, SLB1, and SLB2 vectors are depicted with arrows pointing in the direction of maximal applied force. Copyright The Ohio State University Wexner Medical Center. Published with permission.

men, PJ motion and intradiscal pressure analysis were performed under specific loads in different directions.

\section{Motion Analysis}

Segmental motion assessments were performed at the T7-8 to L1-2 levels for all 5 cycles (INT, PSF, MT, SLB1, and SLB2). The results for PSF, MT, SLB1, and SLB2 are shown as percentages of segmental INT motion in Figs. 3-6 and Table 1 . The range of motion at T9-10 (UIV+1) for each hybrid construct (MT, SLB1, and SLB2) was assessed separately for significant differences in comparison to the PSF construct. Significant motion restriction for SLB1 and SLB2 was noted at T9-10 in flexion (for SLB1 and SLB2, respectively: median $44.9 \%$, IQR $34.7 \%-65.5 \%, \mathrm{p}=0.01$;

\section{Flexion}

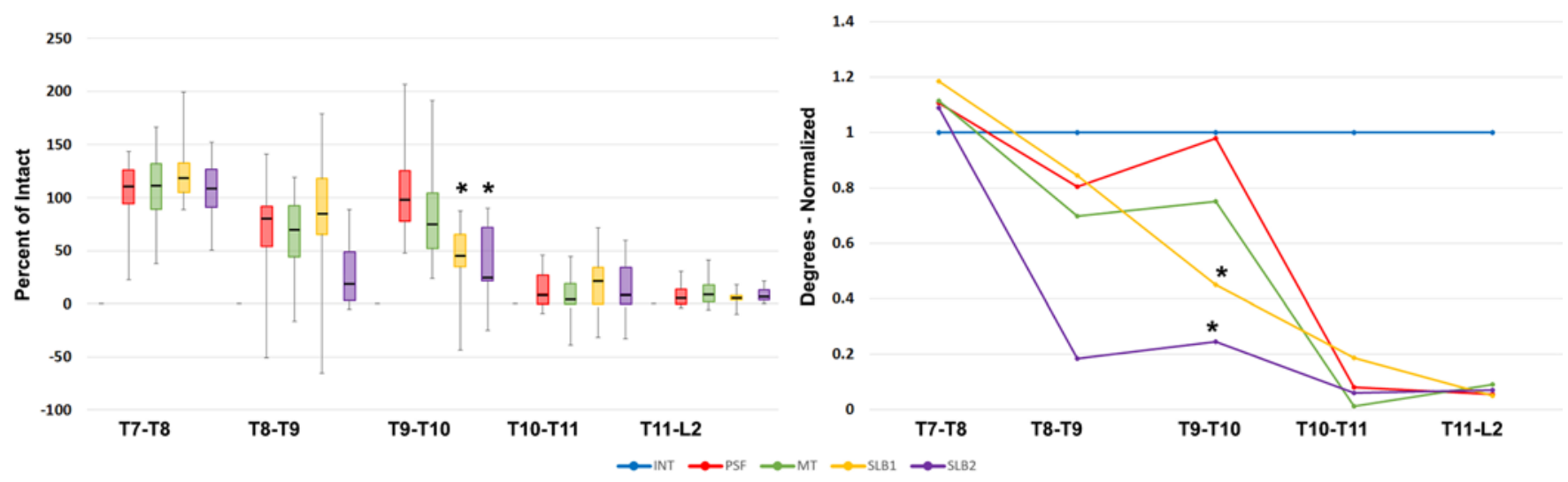

FIG. 3. Only SLB1 and SLB2 show reduced motion at T9-10 as compared to PSF in flexion. Left: Box plots showing median (bold line inside box) percentage of intact flexion motion values at the T7-8, T8-9, T9-10, T10-11, and T11-L2 levels for PSF and the 3 hybrid constructs (MT, SLB1, and SLB2). The boxes indicate IQRs and the error bars indicate the minimum and maximum values. Right: Line graphs of flexion (in normalized degrees) showing a less abrupt transition (reduced slope) between the fixed and mobile transitional segments for the SLB1 and SLB2 constructs. 


\section{Extension}

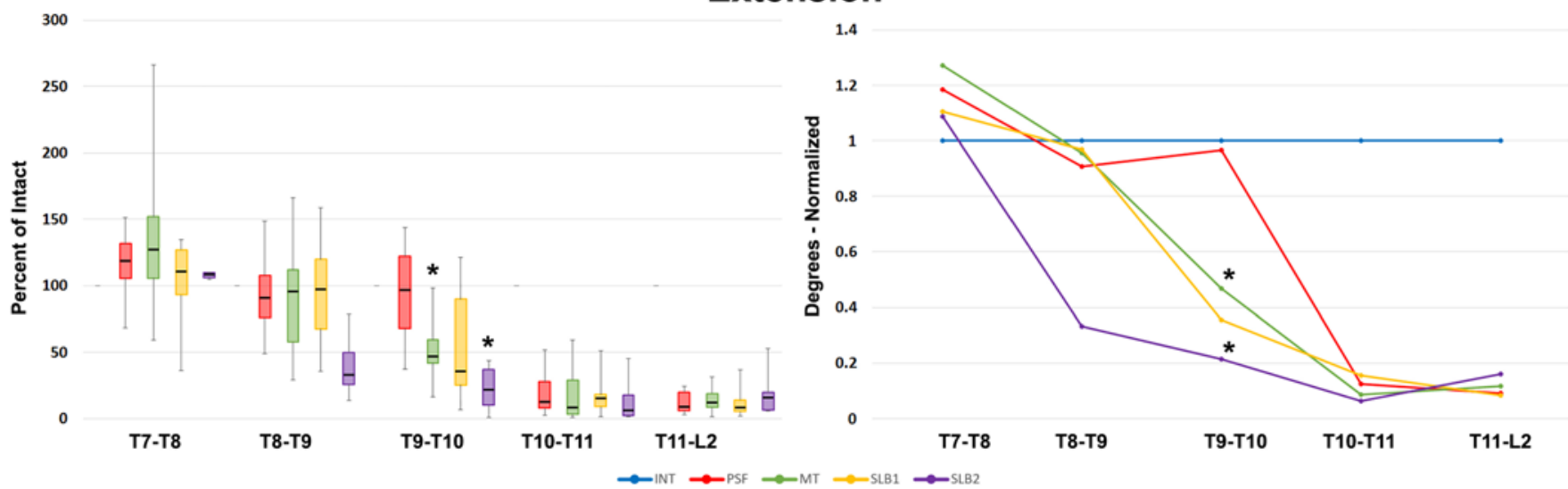

FIG. 4. Only MT and SLB2 show reduced motion at T9-10 as compared to PSF in extension. Left: Box plots showing median (bold line inside box) percentage of intact extension motion values at the T7-8, T8-9, T9-10, T10-11, and T11-L2 levels for PSF and the 3 hybrid constructs (MT, SLB1, and SLB2). The boxes indicate IQRs and the error bars indicate the minimum and maximum values. Right: Line graphs of extension (in normalized degrees) showing a less abrupt transition (reduced slope) between the fixed and mobile transitional segments for the MT and SLB2 constructs.

24.4\%, IQR 21.6\%-72.2\%, $\mathrm{p}=0.02$ ), LB (median 76.4\%, IQR $57.4 \%-80.6 \%, \mathrm{p}=0.003 ; 41.6 \%$, IQR $34.1 \%-46.1 \%$, $\mathrm{p}=0.02$ ) and torsion (median $43.3 \%$, IQR $28.3 \%-48.3 \%$, $\mathrm{p}=0.003 ; 34.3 \%, \mathrm{IQR} 26.0 \%-37.0 \%, \mathrm{p}=0.02)$. Further, SLB2 significantly restricted motion at UIV+1 in extension (median $21.5 \%$, IQR $10.0 \%-36.9 \%, p=0.04$ ). In contrast, MT insertion resulted in a significant reduction in extension only at UIV+1 (median 46.9\%, IQR 41.8\%-59.4\%, p $=0.02$ ).

Range of motion restriction at T8-9 (UIV+2) and at T10-11 (UIV) for each hybrid construct was similarly assessed separately in comparison to the PSF construct (Figs. 3-6 and Table 1). At UIV+2, only SLB2 demonstrated significant motion restriction under moments of lateral bend- ing (median 78.6\%, IQR 71.2\%-83.4\%, $\mathrm{p}=0.03$ ) and torsion (median 48.6\%, IQR 46.6\%-65.5\%, $\mathrm{p}=0.02$ ). Trends toward motion restriction for SLB2 were also noted in flexion (median $18.5 \%$, IQR 3.4-48.8\%, $\mathrm{p}=0.4$ ) and extension (median $33.1 \%$, IQR $25.9 \%-50.0 \%, \mathrm{p}=0.06$ ) but failed to achieve statistical significance. At UIV, SLB2 significantly restricted motion in LB (median $13.1 \%$, IQR $6.4 \%-17.6 \%$, $\mathrm{p}=0.02$ ). No restriction of motion at either T $8-9$ or T10-11 was noted with the MT and SLB1 hybrid constructs.

\section{Intradiscal Pressure Analysis}

Measurements of intradiscal pressure changes $(\triangle \mathrm{IDP})$ were performed at the T8-9, T9-10, and T10-11 intervertebral levels for each of the 5 cycles (INT, PSF, MT, SLB1,

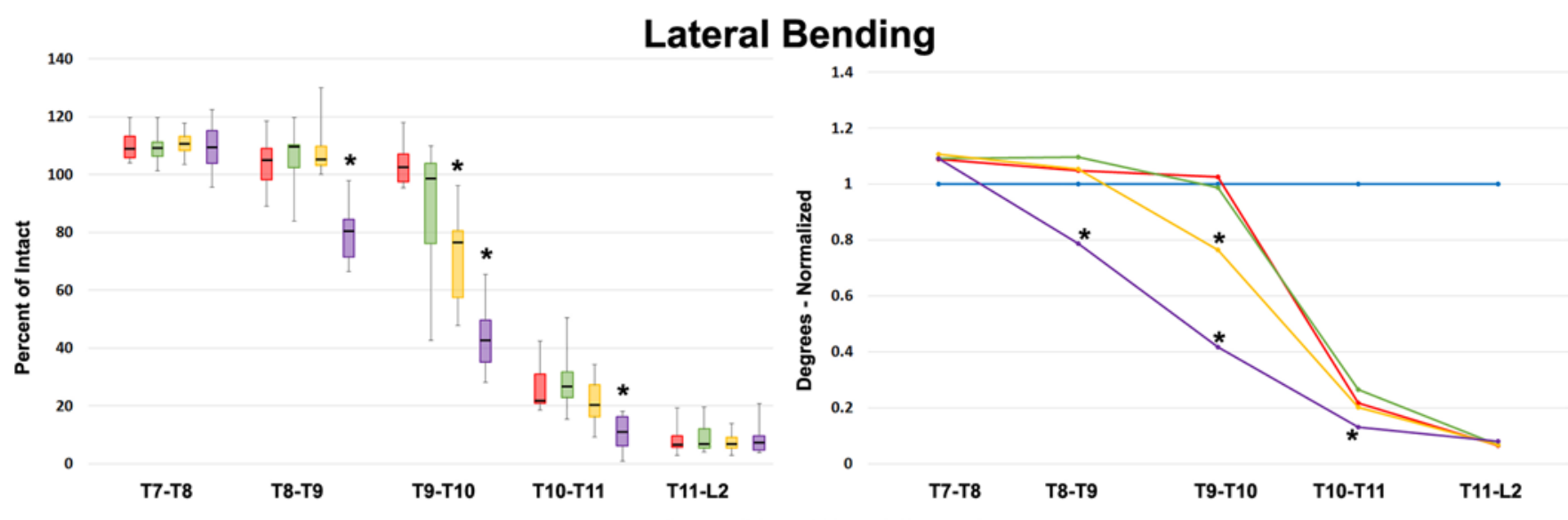

FIG. 5. Only SLB1 and SLB2 show reduced motion at T9-10 as compared to PSF in lateral bending. SLB2 further reduces motion in LB at T8-9 and T10-11. Left: Box plots showing median (bold line inside box) percentage of intact lateral bending (LB) motion values at the T7-8, T8-9, T9-10, T10-11, and T11-L2 levels for PSF and the 2 hybrid constructs (MT, SLB1, and SLB2). The boxes indicate IQRs and the error bars indicate the minimum and maximum values. Right: Line graphs of LB (in normalized degrees) showing a less abrupt transition (reduced slope) between the fixed and mobile transitional segments for the SLB1 and in particular SLB2 constructs. 


\section{Torsion}

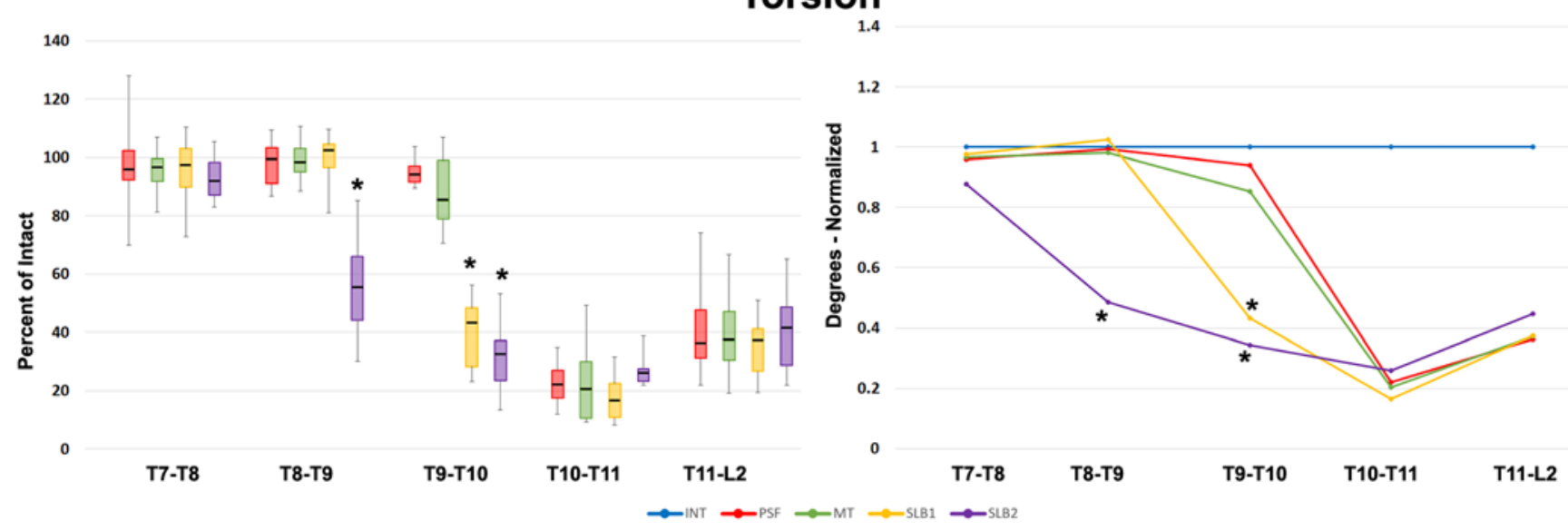

FIG. 6. Only SLB1 and SLB2 show reduced motion at T9-10 and SLB2 further shows reduced motion at T8-9 as compared to PSF in torsion. Left: Box plots showing median (bold line inside box) percentage of intact torsion motion values at the T7-8, T8-9, T9-10, T10-11, and T11-L2 levels for PSF and the 3 hybrid constructs (MT, SLB1, and SLB2). The boxes indicate IQRs and the error bars indicate the minimum and maximum values. Right: Line graphs of torsion (in normalized degrees) showing a less abrupt transition (reduced slope) between the fixed and mobile transitional segments for the SLB1 and in particular SLB2 constructs.

and SLB2; Fig. 7). $\triangle \mathrm{IDP}$ at these intervertebral levels was compared to PSF for each hybrid construct. All 3 hybrid constructs significantly reduced $\triangle \mathrm{IDP}$ at UIV+1 in flexion (MT $-22.3 \%$, IQR $-111.9 \%$ to $18.8 \%, \mathrm{p}=0.01$; SLB1 $57.2 \%$, IQR 20.8\%-73.3\%, $\mathrm{p}=0.01$; SLB2 26.8\%, IQR $-20.3 \%$ to $42.1 \%, \mathrm{p}=0.02$ ) and torsion (MT $66.3 \%$, IQR
$37.8 \%-75.6 \%, \mathrm{p}=0.03 ;$ SLB1 $25.9 \%$, IQR $18.6 \%-40.5 \%, \mathrm{p}$ $=0.02$; SLB2 $22.4 \%$, IQR $12.4 \%-32.8 \%, \mathrm{p}=0.05$ ). Moreover, while at UIV+1, SLB1 and SLB2 significantly reduced $\triangle$ IDP in extension (SLB1 38.4\%, IQR 18.0\%-57.4\%, $\mathrm{p}=0.01 ; \mathrm{SLB} 2-0.9 \%$, IQR $-79.1 \%$ to $13.1 \%, \mathrm{p}=0.02$ ), only SLB2 significantly reduced $\triangle \mathrm{IDP}$ in LB $(32.0 \%$, IQR

TABLE 1. Motion analysis

\begin{tabular}{|c|c|c|c|c|c|c|}
\hline & \multicolumn{2}{|l|}{ T8-9 } & \multicolumn{2}{|l|}{ T9-10 } & \multicolumn{2}{|c|}{ T10-11 } \\
\hline & $\%$ of INT & $p$ Value & $\%$ of INT & $p$ Value & $\%$ of INT & $p$ Value \\
\hline \multicolumn{7}{|l|}{ Flexion } \\
\hline PSF & $80.5(52.3-92.0)$ & 0.057 & $97.9(73.6-132.3)$ & $>0.99$ & $8.0(-0.8$ to 26.2$)$ & 0.003 \\
\hline MT & $69.7(43.2-100.0)$ & $>0.99$ & $75.0(48.2-111.8)$ & 0.837 & 1.1 (-7.8 to 24.2$)$ & $>0.99$ \\
\hline SLB1 & $84.5(56.6-127.3)$ & $>0.99$ & $44.9(34.7-65.5)$ & 0.012 & $18.7(-7.6$ to 34.2$)$ & $>0.99$ \\
\hline SLB2 & $18.5(3.4-48.8)$ & 0.417 & $24.4(21.6-72.2)$ & 0.024 & $6.1(-5.4$ to 32.0$)$ & $>0.99$ \\
\hline \multicolumn{7}{|c|}{ Extension } \\
\hline PSF & 90.7 (75.8-125.7) & $>0.99$ & 96.6 (58.6-124.3) & $>0.99$ & $12.6(5.6-27.8)$ & 0.003 \\
\hline MT & 95.6 (54.9-116.7) & $>0.99$ & $46.9(41.8-59.4)$ & 0.054 & $8.6(2.7-30.6)$ & $>0.99$ \\
\hline SLB1 & $96.9(66.4-126.8)$ & $>0.99$ & $35.7(25.0-94.8)$ & 0.180 & $15.6(7.6-20.3)$ & $>0.99$ \\
\hline SLB2 & $33.1(25.9-50.0)$ & 0.063 & $21.5(10.0-36.9)$ & 0.040 & $6.4(2.4-30.0)$ & $>0.99$ \\
\hline \multicolumn{7}{|c|}{ Lateral bending } \\
\hline PSF & $104.9(98.1-110.2)$ & 0.192 & $102.6(97.5-107.2)$ & 0.588 & $21.7(20.8-31.2)$ & 0.003 \\
\hline MT & $109.6(100.2-110.8)$ & $>0.99$ & 98.7 (68.3-103.9) & 0.138 & $26.6(22.6-32.4)$ & 0.057 \\
\hline SLB1 & $105.2(102.7-110.5)$ & 0.627 & $76.4(57.4-80.6)$ & 0.003 & $20.2(13.7-28.8)$ & 0.099 \\
\hline SLB2 & $78.6(71.2-83.4)$ & 0.033 & $41.6(34.1-46.1)$ & 0.024 & $13.1(6.4-17.6)$ & 0.024 \\
\hline \multicolumn{7}{|l|}{ Torsion } \\
\hline PSF & $99.3(90.7-103.5)$ & 0.933 & $94.1(91.0-97.8)$ & 0.015 & $22.1(17.1-27.2)$ & 0.006 \\
\hline MT & $98.2(94.1-104.6)$ & $>0.99$ & $85.3(77.7-1.00)$ & 0.117 & $20.4(10.6-30.5)$ & $>0.99$ \\
\hline SLB1 & 102.5 (95.2-105.9) & $>0.99$ & $43.3(28.3-48.3)$ & 0.003 & $16.7(10.4-23.5)$ & 0.297 \\
\hline SLB2 & $48.6(46.6-65.5)$ & 0.024 & $34.3(26.0-37.0)$ & 0.024 & $26.0(23.3-28.2)$ & 0.528 \\
\hline
\end{tabular}

INT = intact; PSF = pedicle screw fixation; MT = Mersilene tape; SLB1 = sublaminar band at UIV+1; SLB2 = sublaminar band at UIV+1 and UIV+2.

Data are presented as median (IQR). Boldface type indicates statistical significance. 

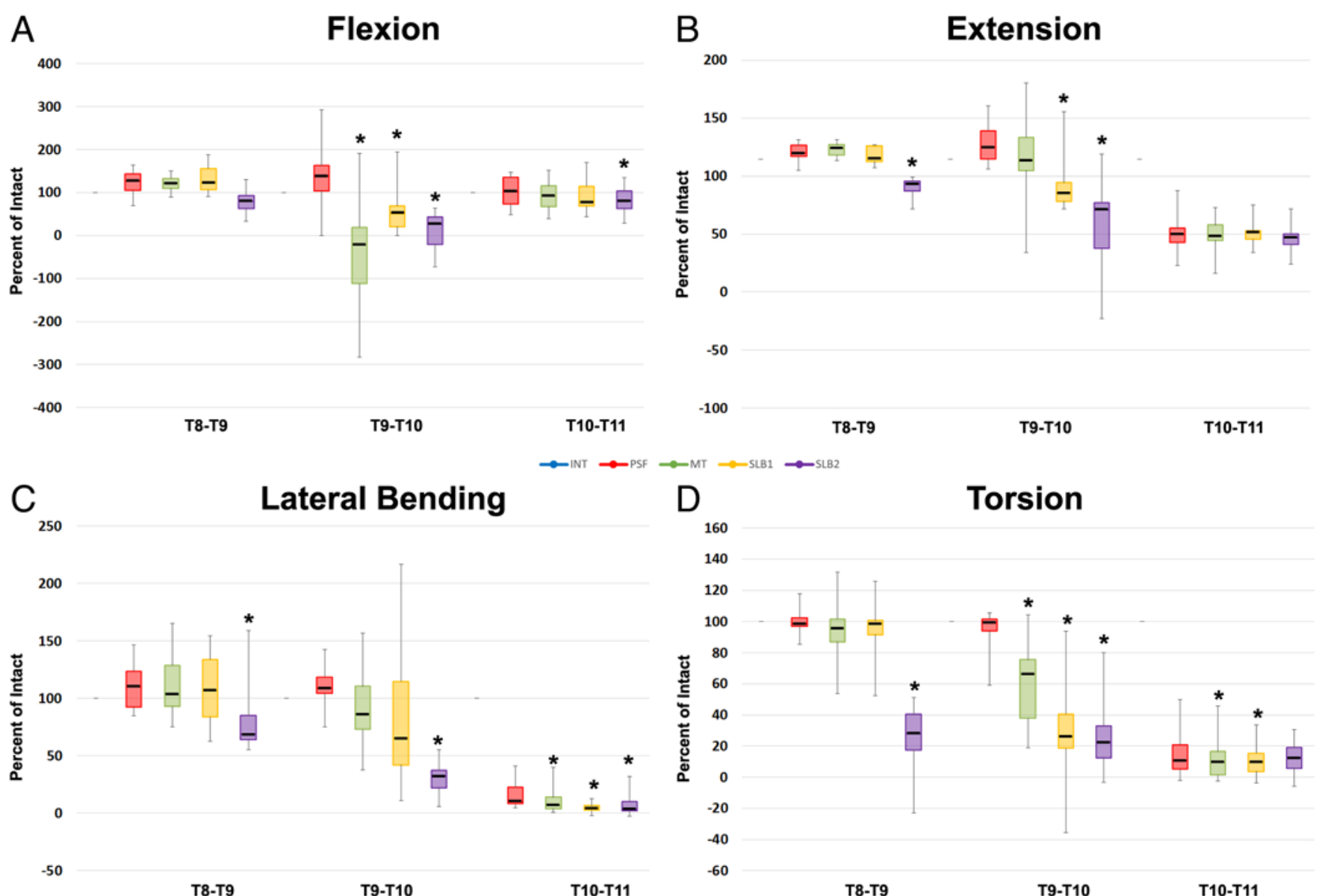

FIG. 7. All 3 hybrid constructs show reduced $\triangle I D P$ in flexion and torsion at T9-10 as compared to PSF. SLB1 and SLB2 reduced $\triangle I D P$ at T9-10 in extension and SLB2 reduced $\triangle I D P$ in lateral bending (LB). SLB2 further reduced $\triangle I D P$ at T8-9 in extension, LB, and torsion. Box plots showing median (bold line inside box) percentage of intact flexion (A), extension (B), LB (C), and torsion (D) $\triangle$ IDP values at the T8-9, T9-10, and T10-11 levels for PSF and the 3 hybrid constructs (MT, SLB1, and SLB2). The boxes indicate IQRs and the error bars indicate the minimum and maximum values.

$21.8 \%-37.1 \%, \mathrm{p}=0.05)$. Finally, at UIV+2, only SLB2 significantly reduced $\triangle \mathrm{IDP}$ in extension $(53.2 \%$, IQR $45.6 \%-$ $57.4 \%, \mathrm{p}=0.05), \mathrm{LB}(68.4 \%$, IQR $63.6 \%-84.9 \%, \mathrm{p}=0.05)$ and torsion $(28.1 \%$, IQR $17.5 \%-40.4 \%, \mathrm{p}=0.04)$.

\section{Discussion}

\section{PJK and PJF Development}

Enhanced quality of life and functional outcome measures following ASD surgery are directly related to the achievement of optimal thoracolumbar alignment. ${ }^{6,25}$ However, the restoration of ideal sagittal balance following long-segment thoracolumbar spinal fusion is associated with increased biomechanical stress at the PJ levels, which is directly implicated in the development of PJK and PJF. $12,15,17$

Various global factors (including low BMD, older age, obesity, under- or overcorrection of pelvic or global sagittal alignment, the type/severity of underlying deformity, and underlying neuromuscular pathologies) and local junctional factors (including disruption of the posterior tension band, use of rigid instrumentation, improper end- vertebra selection, and pre-existing proximal level disc degeneration) have been linked to the development of PJK and PJF. ${ }^{1,9,14,15,17,27-29}$ These factors likely further influence the abrupt changes in stress across the rigid instrumented and mobile non-fused segments of the spine.

Multiple hybrid constructs have been proposed to assist in transitional zone widening at the proximal end of long-segment constructs to reduce the strain placed on the adjacent motion segments. . $^{10,12,17,30}$ Recently, our clinical experience with sublaminar banding at the proximal end of long-segment thoracolumbar spinal fusion constructs to expand the transition zone has been associated with lower rates of PJK and prevention of PJF. ${ }^{27}$ However, no comparative biomechanical studies have been undertaken to date to assess the potential advantage of such semi-rigid hybrid designs to mitigate PJ stress.

\section{Preclinical PJK Studies}

Finite element modeling studies have shown that significant abnormal biomechanical forces are present across the proximal end of long-segment fusion constructs. These pathologic forces result in increases in angular displace- 
ment and intradiscal pressure that lead to PJK. ${ }^{2-4}$ Based on these studies, local biomechanical factors have been identified that can favorably influence these parameters. These factors include preservation of soft tissue elements, the presence of an anchor at the UIV, and the use of a transitional rod, as well as rod curvature and diameter. While supplemental transverse process hooks have not been shown to delay PJF in cadaveric biomechanical testing as compared to pedicle screw-only constructs, ${ }^{17}$ a more gradual transition has been demonstrated over the PJ levels when bilateral hooks replace bilateral UIV pedicle screws with an associated reduction in supra-adjacent segment hypermobility. ${ }^{18,26}$ Further, both PJ range of motion and intradiscal pressures were progressively reduced with increasing numbers of rostrally positioned posterior tethers in a recent long-segment finite element analysis. ${ }^{2}$ Taken together, these studies confirm that rigid UIV fixation causes a significant stiffness differential at the PJ transition zone and that hybrid semi-rigid construct designs could counteract this biomechanical risk factor.

\section{Current Study-Biomechanical Analysis}

Biomechanical studies typically include assessments of load-displacement behavior of one or more functional spinal units (FSUs) under specific conditions. ${ }^{8,13,21}$ The standard universal protocols include range of motion, stiffness, and hybrid testing. ${ }^{13,21}$ The current study design mimics these protocols and prior PJK cadaveric studies in that specimens were unconstrained, with the magnitude and orientation of moments remaining the same with respect to the end vertebra. ${ }^{18}$ Previous studies have demonstrated reduced flexion stability, increased angular displacement, and an increase in intradiscal pressures at the levels proximal to the UIV secondary to instrumented fixation. Such biomechanical alterations have been shown to be further aggravated by the removal of supraspinous and interspinous ligaments. ${ }^{2,3}$

MT restricted motion only under extension loads and reduced $\triangle$ IDP only under flexion and torsion moments at the UIV+1 level. As shown in Fig. 2, MT fastening results in a rostrocaudal force vector. If sufficiently taut, the PJ levels are brought into extension, thus leaving less available "reserve" for further movement, specifically in this direction. In contrast, sublaminar band placement resulted in more consistent PJ motion and $\triangle \mathrm{IDP}$ reductions under loads applied in a majority of directions. Specifically, SLB1 reduced motion in all directions except in extension at UIV+1, and SLB2 was the only hybrid design to reduce motion in all directions at UIV+1 and to reduce motion at $\mathrm{UIV}+2$, both in LB and torsion. Further, at the UIV, SLB2 restricted motion in $\mathrm{LB}$, while no other construct offered any significant reduction in motion at this level. $\triangle$ IDP reductions were seen with SLB1 at UIV+1 (in all directions except LB) and with SLB2 at UIV+1 (in all directions) and $\mathrm{UIV+2}$ (in all directions except for flexion). SLB2 appears to most optimally distribute stresses across the UIV+2 and UIV+1 PJ levels, thereby widening the transitional zone. The observed enhanced effects of banding at UIV+1 and $\mathrm{UIV}+2$ are consistent with the finite element analysis of Bess and colleagues, ${ }^{2}$ where multilevel posterior tethers progressively reduced PJ stresses.
The relative efficacy of sublaminar banding in mitigating PJ stresses relative to MT application appears likely due to the direction and magnitude of the applied force vectors (Fig. 2). While MT is anchored to a transverse connector at the UIV and offers a direct downward pull on the UIV+1 spinous process, sublaminar bands are tightly tensioned at the UIV+1 lamina in a direct anterior-to-posterior direction. This pull most effectively counteracts the post-instrumentation forward angular displacement force on the spine, which directly leads to PJK and PJF. Further, bilaterally positioned sublaminar bands provide greater stress shielding (as compared to a single MT) and render the proximal FSUs biomechanically more stable under physiological loads in various directions. Finally, laminae may represent more reliable anchors in osteoporotic spines, which are more prone to develop PJK and PJF.14

\section{Conclusions}

As compared to MT, sublaminar band placement yields maximal motion restriction and $\triangle$ IDP reduction at the proximal end of long-segment thoracolumbar constructs. Specifically, the use of sublaminar bands at 2 levels allows for the most gradual distribution of forces across the transitional segments.

\section{References}

1. Bess RS, Lenke LG, Bridwell KH, Cheh G, Mandel S, Sides $\mathrm{B}$ : Comparison of thoracic pedicle screw to hook instrumentation for the treatment of adult spinal deformity. Spine (Phila Pa 1976) 32:555-561, 2007

2. Bess S, Harris JE, Turner AW, LaFage V, Smith JS, Shaffrey CI, et al: The effect of posterior polyester tethers on the biomechanics of proximal junctional kyphosis: a finite element analysis. J Neurosurg Spine 26:125-133, 2017

3. Cahill PJ, Wang W, Asghar J, Booker R, Betz RR, Ramsey C, et al: The use of a transition rod may prevent proximal junctional kyphosis in the thoracic spine after scoliosis surgery: a finite element analysis. Spine (Phila Pa 1976) 37:E687E695, 2012

4. Cammarata M, Aubin CE, Wang X, Mac-Thiong JM: Biomechanical risk factors for proximal junctional kyphosis: a detailed numerical analysis of surgical instrumentation variables. Spine (Phila Pa 1976) 39:E500-E507, 2014

5. Cammarata M, Wang X, Mac-Thiong JM, Ce A: Biomechanical analysis of proximal junctional kyphosis: preliminary results. Stud Health Technol Inform 176:299-302, 2012

6. Glassman SD, Berven S, Bridwell K, Horton W, Dimar JR: Correlation of radiographic parameters and clinical symptoms in adult scoliosis. Spine (Phila Pa 1976) 30:682-688, 2005

7. Glattes RC, Bridwell KH, Lenke LG, Kim YJ, Rinella A, Edwards C II: Proximal junctional kyphosis in adult spinal deformity following long instrumented posterior spinal fusion: incidence, outcomes, and risk factor analysis. Spine (Phila Pa 1976) 30:1643-1649, 2005

8. Gonzalez-Blohm SA, Doulgeris JJ, Lee WE III, Shea TM, Aghayev K, Vrionis FD: The current testing protocols for biomechanical evaluation of lumbar spinal implants in laboratory setting: a review of the literature. Biomed Res Int 2015:506181, 2015

9. Hart R, McCarthy I, O’Brien M, Bess S, Line B, Adjei OB, et al: Identification of decision criteria for revision surgery among patients with proximal junctional failure after surgi- 
cal treatment of spinal deformity. Spine (Phila Pa 1976) 38:E1223-E1227, 2013

10. Hassanzadeh H, Gupta S, Jain A, El Dafrawy MH, Skolasky RL, Kebaish KM: Type of anchor at the proximal fusion level has a significant effect on the incidence of proximal junctional kyphosis and outcome in adults after long posterior spinal fusion. Spine Deform 1:299-305, 2013

11. Hostin R, McCarthy I, O’Brien M, Bess S, Line B, BoachieAdjei $\mathrm{O}$, et al: Incidence, mode, and location of acute proximal junctional failures after surgical treatment of adult spinal deformity. Spine (Phila Pa 1976) 38:1008-1015, 2013

12. Kebaish KM, Martin CT, O’Brien JR, LaMotta IE, Voros GD, Belkoff SM: Use of vertebroplasty to prevent proximal junctional fractures in adult deformity surgery: a biomechanical cadaveric study. Spine J 13:1897-1903, 2013

13. Kettler A, Wilke HJ, Haid C, Claes L: Effects of specimen length on the monosegmental motion behavior of the lumbar spine. Spine (Phila Pa 1976) 25:543-550, 2000

14. Kim HJ, Lenke LG, Shaffrey CI, Van Alstyne EM, Skelly AC: Proximal junctional kyphosis as a distinct form of adjacent segment pathology after spinal deformity surgery: a systematic review. Spine (Phila Pa 1976) 37 (22 Suppl):S144S164, 2012

15. Lee J, Park YS: Proximal junctional kyphosis: diagnosis, pathogenesis, and treatment. Asian Spine J 10:593-600, 2016

16. Liu FY, Wang T, Yang SD, Wang H, Yang DL, Ding WY: Incidence and risk factors for proximal junctional kyphosis: a meta-analysis. Eur Spine J 25:2376-2383, 2016

17. Mac-Thiong JM, Levasseur A, Parent S, Petit Y: The influence of proximal anchors on the risk of proximal junctional fracture in the osteoporotic spine: biomechanical comparison between pedicle screws and transverse process hooks. J Spinal Disord Tech 27:E49-E54, 2014

18. Metzger MF, Robinson ST, Svet MT, Liu JC, Acosta FL: Biomechanical analysis of the proximal adjacent segment after multilevel instrumentation of the thoracic spine: do hooks ease the transition? Global Spine J 6:335-343, 2016

19. Panjabi MM: Biomechanical evaluation of spinal fixation devices: I. A conceptual framework. Spine (Phila Pa 1976) 13:1129-1134, 1988

20. Panjabi MM: Hybrid multidirectional test method to evaluate spinal adjacent-level effects. Clin Biomech (Bristol, Avon) 22:257-265, 2007

21. Panjabi MM, Brand RA Jr, White AA III: Mechanical properties of the human thoracic spine as shown by threedimensional load-displacement curves. J Bone Joint Surg Am 58:642-652, 1976

22. Panjabi MM, Goel VK, Takata K: Physiologic strains in the lumbar spinal ligaments. An in vitro biomechanical study 1981 Volvo Award in Biomechanics. Spine (Phila Pa 1976) 7:192-203, 1982

23. Rodriguez-Martinez NG, Savardekar A, Nottmeier EW, Pirris S, Reyes PM, Newcomb AG, et al: Biomechanics of transvertebral screw fixation in the thoracic spine: an in vitro study. J Neurosurg Spine 25:187-192, 2016

24. Schairer WW, Carrer A, Deviren V, Hu SS, Takemoto S, Mummaneni $\mathrm{P}$, et al: Hospital readmission after spine fusion for adult spinal deformity. Spine (Phila Pa 1976) 38:16811689,2013
25. Smith JS, Lafage V, Shaffrey CI, Schwab F, Lafage R, Hostin R, et al: Outcomes of operative and nonoperative treatment for adult spinal deformity: a prospective, multicenter, propensity-matched cohort assessment with minimum 2-year follow-up. Neurosurgery 78:851-861, 2016

26. Thawrani DP, Glos DL, Coombs MT, Bylski-Austrow DI, Sturm PF: Transverse process hooks at upper instrumented vertebra provide more gradual motion transition than pedicle screws. Spine (Phila Pa 1976) 39:E826-E832, 2014

27. Viswanathan VK, Kukreja S, Minnema AJ, Farhadi HF: Prospective assessment of the safety and early outcomes of sublaminar band placement for the prevention of proximal junctional kyphosis. J Neurosurg Spine 28:520-531, 2018

28. Watanabe K, Lenke LG, Bridwell KH, Kim YJ, Koester L, Hensley M: Proximal junctional vertebral fracture in adults after spinal deformity surgery using pedicle screw constructs: analysis of morphological features. Spine (Phila Pa 1976) 35:138-145, 2010

29. Yagi M, Akilah KB, Boachie-Adjei O: Incidence, risk factors and classification of proximal junctional kyphosis: surgical outcomes review of adult idiopathic scoliosis. Spine (Phila Pa 1976) 36:E60-E68, 2011

30. Zaghloul KM, Matoian BJ, Denardin NB, Patel VV: Preventing proximal adjacent level kyphosis with strap stabilization. Orthopedics 39:e794-e799, 2016

\section{Disclosures}

The authors report that research support was provided by Implanet America Inc. for the cadaver experiments performed at the University of Iowa and that Implanet had no role in data gathering, analysis, interpretation, or manuscript preparation. Dr. Grossbach reports a consultant relationshp with DePuy Synthes. Dr. Viljoen reports honorarium receipt from DePuy Synthes for speaking at spine deformity courses.

\section{Author Contributions}

Conception and design: Farhadi, Viswanathan, Minnema, DeVries Watson, Fredericks, Grosland, Viljoen. Acquisition of data: Farhadi, Viswanathan, Ganguly, DeVries Watson, Grossbach, Viljoen. Analysis and interpretation of data: Farhadi, Viswanathan, Ganguly, Minnema, DeVries Watson, Grosland, Viljoen. Drafting the article: Farhadi, Viswanathan, Ganguly, Minnema. Critically revising the article: Farhadi, Viswanathan, Minnema, DeVries Watson, Grosland, Grossbach, Viljoen. Reviewed submitted version of manuscript: all authors. Approved the final version of the manuscript on behalf of all authors: Farhadi. Statistical analysis: Farhadi, Minnema. Administrative/technical/material support: Farhadi, Minnema, DeVries Watson, Grosland, Fredericks, Grossbach, Viljoen. Study supervision: Farhadi, Minnema, DeVries Watson, Fredericks, Viljoen.

\section{Correspondence}

H. Francis Farhadi: The Ohio State University Wexner Medical Center, Columbus, OH. francis.farhadi@osumc.edu. 